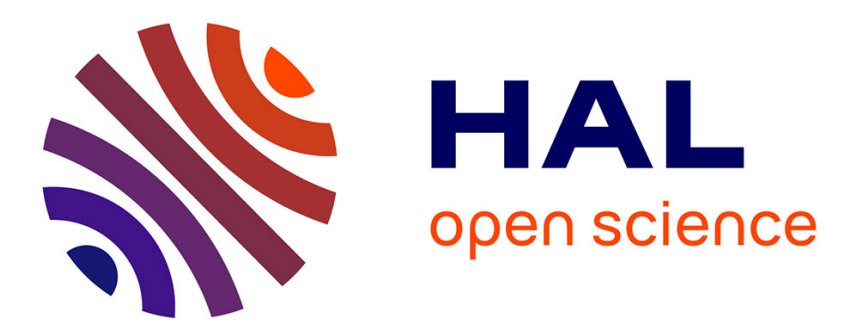

\title{
Contamination du niobium en oxygène au cours de traitements de polissage électrolytique et de recuit sous vide
}

\author{
G. Beranger, P. Boisot, P. Lacombe, G. Amsel, D. David
}

\section{- To cite this version:}

G. Beranger, P. Boisot, P. Lacombe, G. Amsel, D. David. Contamination du niobium en oxygène au cours de traitements de polissage électrolytique et de recuit sous vide. Revue de Physique Appliquée, 1970, 5 (3), pp.383-386. 10.1051/rphysap:0197000503038300 . jpa-00243405

\section{HAL Id: jpa-00243405 https://hal.science/jpa-00243405}

Submitted on 1 Jan 1970

HAL is a multi-disciplinary open access archive for the deposit and dissemination of scientific research documents, whether they are published or not. The documents may come from teaching and research institutions in France or abroad, or from public or private research centers.
L'archive ouverte pluridisciplinaire HAL, est destinée au dépôt et à la diffusion de documents scientifiques de niveau recherche, publiés ou non, émanant des établissements d'enseignement et de recherche français ou étrangers, des laboratoires publics ou privés. 


\title{
CONTAMINATION DU NIOBIUM EN OXYGËNE AU COURS DE TRAITEMENTS DE POLISSAGE ÉLECTROLYTIQUE ET DE RECUIT SOUS VIDE (*)
}

\author{
par G. BERANGER, P. BOISOT et P. LACOMBE, \\ C. R. M. Ecole des Mines de Paris, \\ et G. AMSEL et D. DAVID, \\ Groupe de Physique des Solides de l'E. N. S.
}

\begin{abstract}
Résumé. - La contamination superficielle du niobium en oxygène au cours de traitements de préparation de surface a été étudiée à l'aide de la méthode de micro-analyse fondée sur l'utilisation de la réaction nucléaire $\mathrm{O}^{16}(d, p) \mathrm{O}^{17 *}$. Après un polissage électrolytique et (ou) un recuit sous vide, on observe toujours la présence d'un film d'oxyde dont l'épaisseur est d'une soixantaine d'Angströms environ.

De plus, grâce à cette méthode d'analyse, l'oxygène situé dans le film d'oxyde superficiel a pu être séparé de l'oxygène dissous dans le métal sous-jacent. Il est ainsi montré qu'au cours d'un traitement de recuit sous vide, il y a diffusion d'oxygène et donc contamination de la zone sous-jacente à l'oxyde.
\end{abstract}

Abstract. - The superficial contamination of niobium, by oxygen during surface treatements,
has been studied by the micro-analysis method using the $\mathrm{O}^{16}(d, p) \mathrm{O}^{17 *}$ nuclear reaction. After
electrolyticly polishing and (or) annealing under vacuum, a superficial oxide film is always observed,
the thickness of which being around sixty Angströms.
Moreover, this method allows the oxygen situated in the superficial oxide film and the oxygen
dissolved in the underlying metal to be identified separately. We thus have shown that during
annealing under vacuum, oxygen diffusion occurs and the metal beneath the oxide is so contaminated.

Il est maintenant bien établi qu'un métal peut être contaminé par divers éléments au cours de la préparation de son état de surface par traitement thermique ou par polissage. Cet effet est particulièrement net pour les métaux des groupes IV A et $\mathrm{V} \mathrm{A}$, qui sont très avides d'éléments légers et notamment d'oxygène et d'azote. Ainsi, au cours de recherches sur l'oxydation du zirconium et de ses alliages [1], nous avons montré que le polissage chimique de ce métal en bain fluonitrique entraînait la formation en surface d'une mince couche d'oxyde de quelques dizaines d'angströms d'épaisseur et que, lors d'un traitement de recuit sous vide, il y avait diffusion d'oxygène dans le métal, la couche d'oxyde superficiel étant toujours présente après refroidissement de l'échantillon. Cette contamination est localisée à la surface du matériau. Or, l'état de surface des solides affecte considérablement leurs propriétés, la vitesse d'oxydation par exemple [2] [3]. Il est donc nécessaire, pour un métal, de connaître la contamination induite par de tels traitements de préparation.

Nous avons, pour ce faire, utilisé la méthode de microanalyse fondée sur l'observation directe de réactions nucléaires, et nous l'avons appliquée au

(*) Travaux effectués dans le cadre de la RCP $157 \mathrm{du}$ C. N. R. S. et soutenus par la D. R. M. E. dosage de l'oxygène à l'aide de la réaction $\mathrm{O}^{16}(\mathrm{~d}, \mathrm{p})$ $\mathrm{O}^{17 *}$. Cette méthode, décrite en détail antérieurement [4] [5], présente en effet l'avantage de séparer l'effet de l'oxygène situé dans l'oxyde superficiel, de celui de l'oxygène dissous dans le métal sousjacent, dans la mesure où la couche d'oxyde superficiel ne dépasse pas 2 à 3 microns d'épaisseur $[1,5]$.

Les échantillons $\left({ }^{1}\right)$ de niobium examinés ont subi une partie ou la totalité des traitements successifs suivants :

$$
\begin{aligned}
& \text { - polissage électrolytique, } \\
& \text { - recuit sous vide }\left(10^{-5} \text { torr }\right) \text {, } \\
& \text { - polissage électrolytique. }
\end{aligned}
$$

Afin d'envisager l'influence de la nature et de la composition du bain de polissage électrolytique, deux bains différents ont été utilisés :

Bain $n^{0} 1$

- Acide sulfurique : $90 \%$,

- Acide fluorhydrique : $10 \%$.

Bain $n^{\circ} 2$

- Acide lactique $\quad: 60 \%$,

- Acide sulfurique : $20 \%$,

- Acide fluorhydrique : $20 \%$.

(1) Ces échantillons ont été préparés par M. Fries du Laboratoire de Métallurgie de la Faculté des Sciences d'Orsay. 
Les essais ont été réalisés sur des échantillons de deux puretés différentes. Les teneurs en oxygène, $\mathrm{C}_{0}$, étaient de $330 \mathrm{ppm}$ (lot $\mathrm{n}^{\circ} 1$ ) et $130 \mathrm{ppm}$ (lot $\mathrm{n}^{\circ} 2$ ).

Les différents échantillons ainsi préparés, ont alors été soumis aux essais d'analyse. Nous avons représenté sur la figure 1 le spectre d'énergie des protons émis

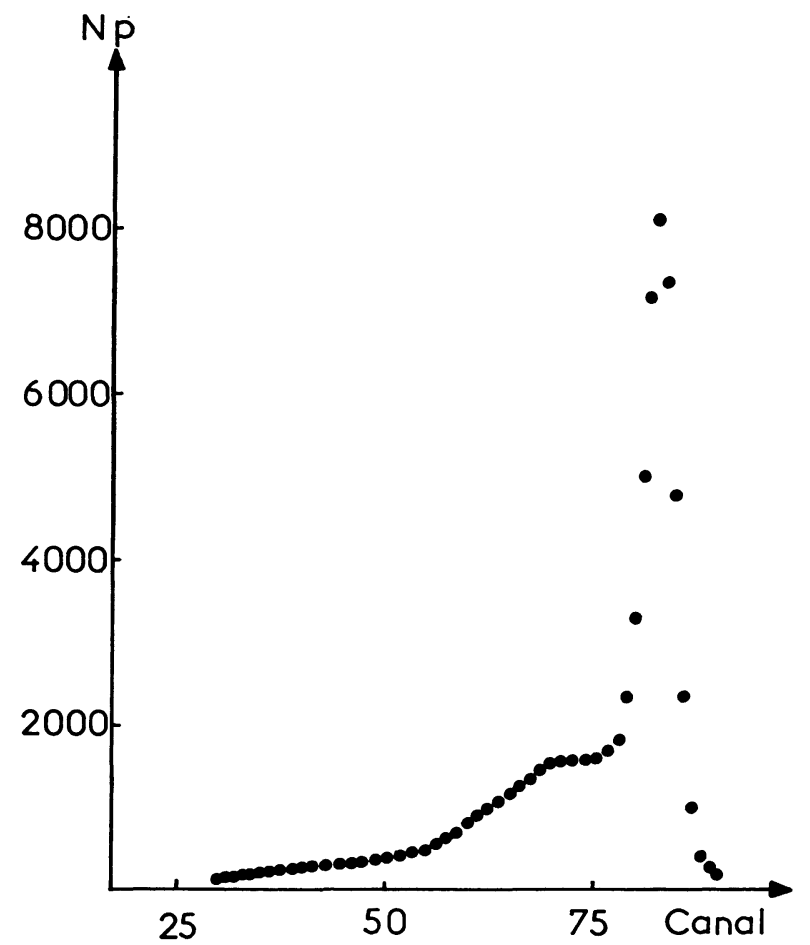

FIG. 1. - Spectre de protons $\mathrm{O}^{16}(\mathrm{~d}, \mathrm{p}) \mathrm{O}^{17} *$ obtenu sur un échantillon de niobium poli électrolytiquement.

au cours de la réaction nucléaire $\mathrm{O}^{16}(\mathrm{~d}, \mathrm{p}) \mathrm{O}^{17 *}$, spectre enregistré sur un échantillon de niobium (lot $n^{0} 1$ ), poli électrolytiquement dans le bain de polissage $\mathrm{n}^{\circ} 1$. Un tel spectre, ainsi que nous l'avons montré antérieurement [1, 5], peut se décomposer en deux parties (Fig. 2):

- La partie I du spectre correspond à l'oxygène situé dans la couche d'oxyde superficiel.

- La partie II correspond à l'oxygène dissous dans le métal, c'est-à-dire la teneur en impuretés oxygène initialement présentes dans le métal, avant tout traitement de préparation de l'état de surface.

Il est possible, à partir d'une telle décomposition, de déterminer le nombre d'atomes d'oxygène correspondant respectivement à la couche superficielle, $31,8 \times 10^{15} \mathrm{At} / \mathrm{cm}^{2}$, et au métal sous-jacent à l'oxyde, $335 \mathrm{ppm}$, valeur en bon accord avec l'analyse initiale du métal.

Les différents résultats concernant les concentrations en oxygène superficiel sont rassemblés dans le tableau I. Nous avons indiqué dans la $5^{\mathrm{e}}$ colonne de ce tableau, une «épaisseur équivalente » d'oxyde. Cette valeur n'est donnée qu'à titre indicatif, pour fixer l'ordre de grandeur. En effet, le calcul a été fait en supposant que la couche superficielle répondait à la

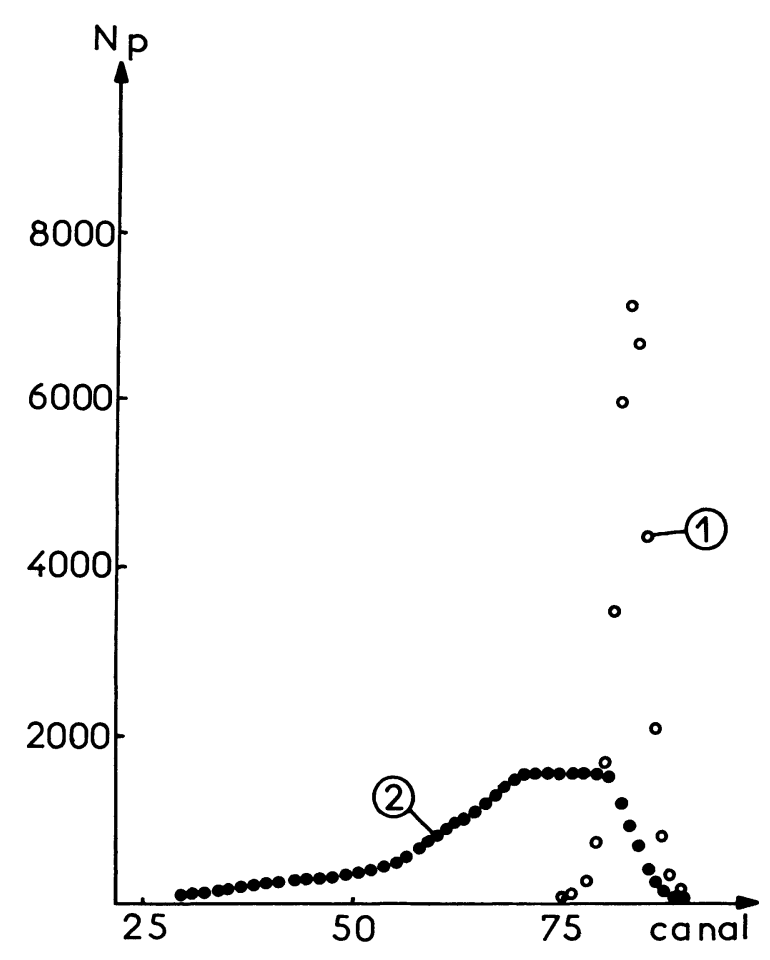

Fig. 2. - Décomposition du spectre de la figure 1. 1) Oxyde de surface.

2) Oxygène dissous dans le niobium.

formule $\mathrm{Nb}_{2} \mathrm{O}_{5}$, la densité de l'oxyde $\mathrm{Nb}_{2} \mathrm{O}_{5}$ étant de 4,6. Ceci n'est qu'une approximation car d'une part on ignore totalement la structure d'un tel film, et d'autre part il est probable que des ions étrangers provenant $\mathrm{du}$ bain électrolytique aient été incorporés au cours du polissage.

On constate que dans tous les cas, il y a présence d'un « film superficiel d'oxyde ». Notons que, dans le cas particulier du niobium, pour les deux bains utilisés, la nature du bain de polissage n'a pas d'influence significative sur l'épaisseur de l'oxyde.

Si l'on compare (Fig. 3) les spectres obtenus sur deux échantillons de niobium, contenant les mêmes impuretés initiales en oxygène $(130 \mathrm{ppm})$ poli électrolytiquement d'une part, poli électrolytiquement puis recuit sous vide secondaire $\left(1 \times 10^{-5}\right.$ torr $)$ pendant 2 heures à $600^{\circ} \mathrm{C}$ d'autre part, on constate que de l'oxygène a diffusé au cours du recuit dans la zone sous-jacente à l'oxyde. Il y a donc lors d'un traitement de recuit, une nette contamination qui s'étend sur une profondeur variable avec la durée et la température $\mathrm{du}$ recuit.

On peut, en comparant les spectres de la figure 3, décomposer (Fig. 4) le spectre obtenu après recuit. On met ainsi en évidence trois parties dans le spectre :

- La partie I qui correspond à l'oxygène situé dans la couche d'oxyde superficiel.

- La partie II qui correspond à l'oxygène présent dans le métal avant le traitement de recuit.

- La partie III qui correspond à l'oxygène ayant diffusé au cours du traitement de recuit. 
d'oxygène ayant diffusé est nettement inférieure à la quantité d'oxygène présente dans la couche d'oxyde superficielle initiale $\left(30 \times 10^{15} \mathrm{At} / \mathrm{cm}^{2}\right)$. Donc dans ces conditions, on devrait avoir dans le métal, à l'interface métal-oxyde, la concentration d'équilibre entre l'oxyde et le métal donnée par le diagramme niobiumoxygène, c'est-à-dire 4,7 At \% La valeur expérimentale est nettement inférieure à cette concentration. Des expériences sont actuellement en cours pour expliquer ce phénomène qui pourrait être dû à de la diffusion par joints de grains, ou à une perturbation superficielle du métal sur quelques microns.

Les résultats obtenus sur le niobium sont tout à fait semblables à ceux obtenus dans le cas du zir- conium [1] et du titane, ce qui confirme l'analogie existant entre ces métaux en ce qui concerne leur avidité vis-à-vis de l'oxygène.

En conclusion, il est nécessaire d'effectuer sur le niobium un traitement de décapage et de polissage qui, d'une part élimine toutes les impuretés présentes à la surface du métal, et d'autre part, donne le film d'oxyde le plus mince possible. De plus, dans les cas où un traitement de recuit est nécessaire, il faut, après ce recuit, effectuer un nouveau polissage : celui-ci permet d'éliminer la zone contaminée au cours du recuit. Mais cependant, après un tel polissage, on observe toujours un film d'oxyde superficiel qui, lui, ne peut être éliminé.

\section{Bibliographie}

[1] Amsel (G.), David (D.), Béranger (G.), Boisot (P.) De Gelas (B.) et Lacombe (P.), J. Nucl. Mat., 1969, 29, 144.

[2] Wallwork (G. R.), Rosa (C. J.) et Smeltzer (W. W.), Corr. Sci., 1965, 5, 113.
[3] Gulbransen (F. A.) et Andrew (F. K.), J. Met., 1957, 9, 394.

[4] Amsel (G.) et Samuel (D.), Anal. Chem., 1967, 39, 1689.

[5] Amsel (G.), Béranger (G.), de Gelas (B.) et Lacombe (P.), J. Appl. Phys., 1968, 39, 2246. 